\title{
Let's not blame the patient: Understanding the benefits and shortcomings of population health in orthopaedic surgery
}

\author{
Chad Amato ${ }^{1}$, Zain Sayeed ${ }^{1,2,3,4}$, Mark Lane ${ }^{1}$, Muhammad T. Padela*1,2,3,4, Enrique Feria-Arias ${ }^{1}$, Sam Nasser ${ }^{1}$, \\ Hussein F. Darwiche ${ }^{1,2}$, Khaled J. Saleh ${ }^{4}$ \\ ${ }^{1}$ Department of Orthopaedic Surgery and Sports Medicine, Detroit Medical Center, Detroit, MI, United States \\ ${ }^{2}$ Resident Research Partnership, Detroit, MI, United States \\ ${ }^{3}$ Department of Orthopaedic Surgery, Chicago Medical School, Rosalind Franklin University, North Chicago, IL, United States \\ ${ }^{4}$ Michigan Musculoskeletal Institute, Madison Heights, MI, United States
}

Received: February 28, 2018

DOI: $10.5430 /$ jha.v7n3p49
Accepted: May 4, 2018

Online Published: June 1, 2018

\begin{abstract}
Population health is a concept that emerged from the desire of providers to care for patients in a manner that produces the best possible outcomes while minimizing cost. It may be defined as the study of medical data of large groups of people in order to recognize and investigate patterns. This information is then used to create disease management guidelines that streamline care and regulate practice patterns. Whereas population health looks to recognize commonalities in data, the concept of patient-centered care focuses on embracing individualization and increasing the involvement of each patient within their treatment planning. Combining both perspectives creates a challenge for providers and patients to strike the proper balance between adhering to standardized guidelines based on the treatment methods and outcomes recognized in populations and applying it clinically to individual patients. A significant contribution of population health studies is the identification of risk factors associated with increased rates of complications following total joint arthroplasty as well as preventative measures for conditions such as osteoarthritis. However, to employ these findings in a patient-centered manner orthopaedic surgeons must take this a step further and also evaluate a patient's ability to adhere to the recommendations by exploring factors such as home environment and socioeconomic factors, thus proactively addressing issues that could hinder patient compliance. With focused collection methods of acquiring data, these two practices of care will hopefully begin to see less divergence when it comes to applying data derived from population health initiatives to individual patients in a patient-centered manner.
\end{abstract}

Key Words: Population health, Value-based medicine, Patient-centered care, Big data

\section{INTRODUCTION}

Healthcare reform has rapidly changed the method by which musculoskeletal care is delivered nationally. Population health and patient-centered care represent two sides of the same token which defines the currency of value-based or- thopaedic care. As healthcare increasingly emphasizes valuebased delivery, the proper application of these concepts remains to be delineated. Population health offers surgeons a tremendous ability to consider how to effectively manage a condition that caters to a local network of patients, and how

\footnotetext{
*Correspondence: Muhammad T. Padela; Email: tpadela@gmail.com; Address: University Health Center, 4201 St. Antoine St., Suite 9B, Detroit, MI, 48201, United States.
} 
prevention may play a larger role in orthopaedic conditions. Intertwining both patient-centered and population health may facilitate improved quality reporting and potentially improve patient outcomes for years to come. Some examples of combining these concepts includes the development of locally specific electronic medical record (EMRs), clinical practice guidelines, and appropriate use criteria.

Within this review we will discuss the concepts of population health and patient-centered care. Ideally, population health and patient-centered care should act synergistically to allow surgeons to provide the best care possible to their patients. However, within the current landscape of the healthcare field these two concepts can instead find themselves in opposition. The barriers to cohesive implementation as well as recommendations to continue to unite these ideals will be discussed here.

\section{Methods}

In order to best characterize the current sentiment regarding the topics of population health and patient centered care (PCC) amongst professionals within the medical field and more specifically the field of orthopaedics, our team sought to review the most recent articles in the medical literature surrounding this topic. The primary electronic database used to identify relevant articles was PubMed and Google Scholar. Utilizing search terms such as "Population Health Orthopaedics", "Patient Centered Care Orthopaedics", "Database Research in Orthopaedics", and "Big Data Medical Research" we were able to compile an initial list of articles of interest. From this initial list we implemented criteria such as limiting included papers as much as possible to those that have been published within the preceding three years so as to present the most up to date information possible. Bibliographies from the resulting list of publications reviewed were further examined to obtain additional sources to gather more data relevant to our review.

\section{RESULTS}

The following will provide an overview and summary of recent published data relevant to the concepts of PCC, Population Health, their role within and on the field of orthopaedic surgery, and relevant topics that comprise these healthcare concepts.

\subsection{Providing value in orthopaedic care}

When we speak of providing high-value care to patients it is important to have an understanding of how the term value is derived. Value equals health outcomes achieved per dollar spent or outcomes/cost. ${ }^{[1]}$ Depending on the perspective that one takes when evaluating treatment outcomes, especially in our discussion of population vs patient based care, this variable drives various metrics. What is ultimately chosen to be included can impact the practice patterns of physicians and their interactions with patients. Outcomes can consist of metrics detailed by government agencies or payers such as length of stay (LOS), infection rates, readmissions, and mortality. ${ }^{[2]}$ In addition, patient reported measures such as pain level, satisfaction with their surgery, lifestyle impairment and/or improvement may also be included. ${ }^{[3]}$ Costs when used in the context of high-value care refers to the entire spectrum of care that a patient receives related to his or her medical condition. ${ }^{[4]}$ An example in orthopaedics may refer to treating a patient with a TJR: the episode of care for this might include the initial office visits, imaging, surgical procedure, hospital stay, and follow up for a determined time interval following surgical intervention. ${ }^{[5]}$

In light of the desire to increasingly focus on providing highvalue care to patients and populations, two concepts emerged that shifted the mindset of providers from volume to value, Population Health and Patient-Centered Care. ${ }^{[6]}$ In an ideal scenario population health and patient-centered care should work hand in hand with one another in order to provide the best care possible to the orthopaedic patient. However, within the current climate of healthcare reform, reporting requirements, quality measure goals, and the imprecise definitions of these terms makes this a challenge to accomplish. ${ }^{[7]} \mathrm{We}$ will clarify what is meant by these terms and later discuss the components of each paradigm and how they can, at times, be in harmony or at odds.

\subsection{Population health vs. patient-centered care from an orthopaedic standpoint}

\subsubsection{Population health}

Population health may be defined as the health outcomes of a group of patients, including the distribution of such outcomes within that group. These groups can be sub-classified by employment, age, race, geography, etc. The outcomes of these groups holds tremendous impact upon policy formation in national and local forums. There are many health determinants that impact population health and are often described as risk factors such as medical care systems, individual behavior, genetics, a social environment, and a physical environment.

\subsubsection{Patient centered care}

PCC is a concept that has been discussed within the healthcare field for several decades, however, a finite definition is still lacking. ${ }^{[8]}$ A recent definition describes patient-centered care as "care that is respectful of and responsive to individual patient preferences, needs, and values, and ensuring that patient values guide all clinical decisions. ${ }^{[9]}$ Research by the Picker Institute has delineated 8 dimensions of patient- 
centered care, including: 1) respect for the patient's values, preferences, and expressed needs; 2) information and education; 3) access to care; 4) emotional support to relieve fear and anxiety; 5) involvement of family and friends; 6) continuity and secure transition between health care settings; 7) physical comfort; and 8) coordination of care. ${ }^{[10]}$ Initiatives such as Patient Centered Medical Homes (PCMH) have been developed in order to meet these dimensions by streamlining care amongst providers and allowing patients to play a larger role in determining their care plan. ${ }^{[11]}$ However, based on this definition of PCC, potential conflict can arise when attempting to merge goals of population health and patient-centered care together.

The concept of population health seeks to obtain information from large segments of a selected group and from this group extract data to evaluate factors related to a given medical conditions. These factors include presenting symptoms, diagnostic studies, approaches to treatment, and treatment outcomes. ${ }^{[12]}$ From this data concepts for disease management can be developed based on common patterns recognized within the data. This information can further be utilized to standardize management of the condition or disease under investigation in order to streamline care and regulate practice patterns. Whereas population health looks to recognize commonalities in data, the concept of patient-centered care focuses on embracing individualization and increasing the involvement of each patient within their treatment planning. ${ }^{[9]}$ The idea that each patient has a unique background and may perceive a diagnosis or recommended course of treatment differently is a central component of the PCC model. The current challenge for providers and patients is to strike the proper balance between adhering to standardized guidelines based on the treatment methods and outcomes recognized in populations and applying it clinically to individual patients.

\subsection{The major difference between patient centered care and population health}

The concept of provider accountability for the health of a large group, or population, is central to alternative payment models advanced by the U.S. Centers for Medicare and Medicaid Services (CMS) such as Accountable Care Organizations (ACOs) and PCMHs that use metrics tracked and benchmarked over large populations. Additionally, related terms such as "population management" and "practice-based population health" are often used in exchangeable fashions when related to newer health policies. The goal of population management is to keep the population of a study as healthy as possible, minimizing the need for expensive interventions such as emergency department visits, hospitalizations, imaging, and procedures.
In order for population health to impact PCC in a positive or negative manner data must be collected in order to properly examine a population of interest. To do this physicians and researchers must have a method to obtain large volumes of information regarding their target population. Ideally this information would be collected from as many members of a selected group as possible. This allows for examination of similarities and differences in care management based on factors such as regional geography, inpatient/outpatient settings, socioeconomic factors, and comorbid conditions. This need for vast amounts of information has led to the rise of "big data" and its increasing role in the medical field, namely in the form of informational databases.

\subsection{Identify the role of big data in healthcare delivery}

To obtain this population health information the healthcare field increasingly relies on the concept of "big data". Big data is a branch of health care informatics that pools large and disparate datasets and applies a suite of mathematical approaches that derives associations, facilitates comparisons, and generates insights that are otherwise not possible using standard mono-source analytics. ${ }^{[13]}$ In order to obtain this data in an organized manner databases and registries such as the CMS or clinical registries such as the American Joint Replacement Registry (AJRR) have been developed to allow for data to be pooled from various sources across the country in a centralized manner. ${ }^{[14]}$ As researchers increasingly use these resources to conduct studies in order to track the value of care being provided, big data can serve to alter practice patterns by way of helping to form clinical guidelines or by influencing the ways in which physicians receive reimbursement. ${ }^{[15,16]}$ If these sources do not encompass data on variables that represent factors important to the orthopaedic patient, a conflict can emerge between patient and surgeon. Providers will find themselves balancing compliance with quality and reporting standards set by population derived data, and merging that with the values and desires of each individual patient.

The role of big data will only continue to grow within the field of orthopaedics as our methods for analyzing the data become more sophisticated. ${ }^{[17]}$ As the rise of big data and database derived research increasingly impacts the field of orthopaedic surgery, it is critical to understand the background of data and the potential limitations of these sources. Researchers and end-users of the data need to be acutely aware of how and what data is collected in these databases in order to apply the information correctly to their studies or practice. ${ }^{[12,18]}$ Naidus and Celi offer the concept of big data leading to "big noise" when not applied and analyzed properly. ${ }^{[19]}$ With the vast amount of data being collected today, 
the risk of discovering unintended and ultimately unrelated correlations can increase and can potentially lead to inappropriate changes in practice patterns if the data comprising these studies is not properly interpreted. ${ }^{[20]}$

The CMS claims database and National Inpatient Sample database (NIS) represent two of the most utilized sources in orthopedic research. ${ }^{[14]}$ In the case of the CMS Medicare claims database, it also serves a purpose to form standards and evaluate compliance for pay-for-performance models. ${ }^{\text {[21] }}$ The CMS Medicare claims database is able to collect information from beneficiaries and track their data through both inpatient and outpatient encounters. While this is useful to track a full episode of care, the data is limited to the analysis of elderly patients and the disabled making generalizability problematic. ${ }^{[12]}$ Another example, the NIS database tracks patient data during the period that patients are treated in the inpatient hospital setting. ${ }^{[12]}$ While this is useful for tracking complication and cost during the inpatient setting, the ability to evaluate these aspects over the entire episode of care is lacking. Both of these sources are heavily reliant on the input of data via coding systems namely, International Classification of Disease (ICD) coding. ICD-9 suffered from lack of specificity in its reporting of medical conditions such as laterality of an injury. With the adoption of ICD-10 coding, greater specificity has been added in order to address some of these concerns. ${ }^{[22]}$ However, lack of specificity remains in this version as well. For example a wrist fracture can be documented with its location and complications such as non-union, however the initial cause of the injury cannot be specified. ${ }^{[23]}$ A fracture resulting from a car accident or from a patient defending themselves from an abusive spouse, for example, greatly impacts the counseling and interaction with that patient as a physician. This highlights another disconnect between data gathered at the population level and applying it to individual patients.

Studies have also shown the potential for misreporting of comorbidities within databases that are then used to examine complications rates. ${ }^{[24]}$ With data errors such as this, one can imagine a scenario where orthopaedic practitioners mistakenly misinform patients regarding their candidacy for surgical procedures based off of data interpretation that may be faulty.

\subsection{Data tracking in population health and patient- centered care}

Healthcare participants in MIPS and APM initiatives must report and comply with quality measures using population health and database driven metrics in order to avoid reimbursement penalties. ${ }^{[25]}$ Using MIPS as an example physicians have the option to choose which quality measures to report and many of these metrics can be achieved and reported without significant input from the patient. ${ }^{[26]}$ One study compared online reviews of orthopaedic surgeons and found that 5 variables were statistically linked to higher ratings. ${ }^{[27]}$ These included ease of scheduling, time spent with patient, wait time, surgeon proficiency/knowledge, and bedside manner. This demonstrates factors that are important to the orthopaedic patient, however, there are currently no databases that routinely record all of these variables. If these databases are the standard to which population health and healthcare reform are derived, this demonstrates a disconnect that exists between the information and policies surgeons are asked to adhere to and the desires of the patient.

\section{DisCUSSION}

\subsection{How has population health contributed to the or- thopaedic field?}

One of the more significant contributions that population health and studies utilizing clinical databases have provided to the orthopaedic field is the identification of risk factors associated with increased rates of complications following TJA as well as preventative measures for conditions such as osteoarthritis. ${ }^{[28]}$ Three significant risk factors identified in the literature for poor outcomes following TJA have been obesity, poorly controlled diabetes, and smoking. ${ }^{[2-31]}$ For chronic disease management, weight loss has been indicated to decrease osteoarthritis; this recommendation comes from a meta-analysis that evaluated various orthopaedic observational studies utilizing database sources. ${ }^{[32]}$ However, to employ this in a patient-centered manner physicians must take this a step further. Surgeons must also evaluate a patient's ability to adhere to this recommendation. By exploring factors such as home environment and socioeconomic factors, surgeons can identify potential barriers to adhering to recommendations such as weight loss. By doing so, orthopaedic surgeons can more effectively translate and apply these population based recommendations to the patients he or she encounters in daily practice by proactively addressing issues that could hinder compliance.

\subsection{What is the role of orthopaedic surgeons in popula- tion health and patient-centered care?}

Approximately 54 million and 27 million Americans are affected by osteoporosis and osteoarthritis respectively. ${ }^{[33,34]}$

These are two conditions that orthopaedic surgeons are positioned to provide input via data provided by the patients they treat and for that data to be translated into recommendations to help the population of people affected by these conditions. One area of data that surgeons are in a prime position to aid in population health initiatives is the Patient Reported Outcome Measures. Patient Reported Outcome Measures or 
PROM's represent what has been termed the "missing link" toward truly understanding patient outcomes. ${ }^{[35]}$ As opposed to measures such as LOS or infection rates, these measures represent the outcomes that patients are experiencing in their daily lives and can help healthcare providers become more in tune with these aspects and how they relate to patient care, such as improvement in function and pain, or level of satisfaction with the procedure and the surgeon who performed it. $^{[36]}$

From a PCC perspective, surgeons in permissible practice settings can become active participants in PCMH initiatives. Through coordinating with PCPs within the PCMH, providers can work together to establish better understanding of TJR indications, quality-of-life improvement, and postop expectations for rehab and pain levels. ${ }^{[37]}$ This can help patients receive more information throughout the process of considering intervention than what they would normally get by talking to the surgeon alone. Incorporating aspects of the patient's health in the reverse direction from PCP to specialist can also take place such as EMR alerts when patients need follow up on lab work related to non-orthopaedic conditions allowing for surgeons to promote preventive measures and promote early intervention. ${ }^{[38]}$ However, in order to accomplish these goals the adoption of EMR and IT resources capable of integrating data provided by patients in the form of PROMs and information from the PCP office in a streamlined and easy to use manner becomes paramount. ${ }^{[39]}$

\subsection{The effect of population health on patient-centered care}

The nature of population health is to organize and form patient groups based on various selected factors. ${ }^{[40]}$ However, this practice can lead to health care providers projecting a preconceived notion regarding members of a given population that patients may become acutely aware of. When patients believe they are being judged prematurely based on issues such as age, race, socioeconomic status, etc. this can negatively impact the physician-patient relationship. Patients may resist following up on planned treatments, rate physicians poorly on evaluations, as well as disregard recommendations for preventative health measures, which is a significant goal of population health initiatives. ${ }^{[41,42]}$

Dr. Hilary Hatch outlines three reasons why patients may not adhere to treatment guidelines based off of population information that may become too formulaic and standardized without taking into account individual patient circumstances. ${ }^{[43]}$ People are keenly aware of being told to do things that are not for their personal benefit. ${ }^{[35]}$ People reject recommendations that do not match their health needs and people are much more likely to follow recommendations from people they

Published by Sciedu Press trust. ${ }^{[11]}$ She points out that the first two reasons undermine patient trust. ${ }^{[43]}$ Eddy et al. argue that an intervention should be classified as the "standard" of care only if there is "virtual unanimity among patients about the overall desirability". ${ }^{[44]}$ This highlights the importance of including patient input through methods such as direct conversation and/or PROMS into the discussion of treatment. It also highlights a need for physicians to increase the level of communication with patients in order for them to understand the benefit when it may not be readily apparent why it affects them. This issue demonstrates again the conflict that providers can face when applying population health standards and recommendations with members of their practice in a patient-centered manner.

Another issue with aligning population health initiatives and patient-centered care is the degree of latitude that physicians have when reporting quality measures for compliance with initiatives such as MIPS. Physicians select six quality measures from a list that includes documentation of perioperative antibiotic prophylaxis, administering venous thromboembolism prophylaxis prior to surgery, and Functional Status Assessment for Total Knee Replacement among others. ${ }^{[26]}$ Even in the case of the functional status assessment all of the orthopaedic specific quality measures for example represent process rather than outcome measures. This creates an incentive to include measures that can be completed quickly and in the same time encounter such as VTE, and antibiotic prophylaxis. While these may satisfy the requirement, they do little to add information on the patient-centered care or population health front. When physicians are faced with already short appointment times the ability to achieve these measures outside of the appointment window can be enticing for practitioners to select. ${ }^{[45]}$

As discussed above, reporting data as well as collecting data increasingly involves utilization of an EMR. However, physicians find themselves spending more time with EMR modalities thus leaving less time to spend with patients and having the proper level of communication that is vital to a strong physician patient relationship. ${ }^{[46]}$ While measures such as communication may not be a required quality measure for physicians to report, it undoubtedly impacts the patient. For example, Goldsmith et al. demonstrated the considerable impact that patient-perceived level of support has on their experience with receiving a TKA procedure. ${ }^{[47]}$ This included informational, clinical, and personal experiences. One of the most striking takeaways from this study is how the role of physician interactions with the patient can impact the overall experience of the operation. This includes taking time to thoroughly explain the procedure during pre-op appointments, approaching interactions with patients in an empathetic manner, and being available to speak with patients regarding post- 
op complications. These factors were viewed so highly by some of the participants in the study such that "even though their TKA outcome was not as good as they expected, the time and information provided by their surgeon both before and after the surgery improved their TKA experience". [47]

Finally, with initiatives such as the "Hospital Compare" website which reports publicly available hospital rankings based on measures, such as rate of complications for hip/knee replacement patients, one can envision the potential for patients being denied a certain procedure based upon data that relates their specific comorbidities or disease process to longer lengths of stay and/or increased rates of complication. This can possibly lead to patients receiving delayed treatment due to fear among physicians for receiving poor ratings. ${ }^{[48,49]}$

\subsection{How can we continue to merge population health and patient-centered care in the future?}

Surgeons are caught in a tough position between contributing to population health measures and aiding in the advancement of the field, while also facing the burden of meeting quality reporting requirements for reimbursement. As detailed in earlier sections, with time crunches in the office setting and increasing EMR demands, reporting measures that may not directly address patient concerns but are more convenient for clinicians may take precedent. One strategy for merging the two philosophies closer is the implementation of reporting requirements that focus more on patient centered measures such as PROMs. Organizations such as the International Society of Arthroplasty Registries (ISAR) are taking steps to give patients a greater voice and a greater share of the data by advocating for the inclusion of PROMs into clinical registries such as the AJRR. ${ }^{[50]}$ While organizations such as CMS are also beginning to track these types of measures and include them within their value-based payment systems, simply adding it as another requirement can have equally detrimental impacts on patient-centered practice if physicians are further burdened by EMR demands and alerts. ${ }^{[51]}$ Simplifying the data reporting metrics to focus on patient centered measures can allow for tracking of data that is more patient-centered and decrease the time burden of physicians with EMR demands, allowing time to be allocated to more face-to-face conversations with patients and increased levels of patient satisfaction. ${ }^{[47]}$ In essence, the aim could be described as collecting and reporting the right data, versus simply more data.

The method of obtaining patient reported data can also impact the success of merging population health and patient centered initiatives. Engaging patients to provide the data is one challenge to be overcome. To do so Dr. Neil Wagle describes three methods for successful data collection, (1) Make it easy: Provide patients with an interface to record data in a convenient way for them. ${ }^{[35]}$ Initiatives such as the app developed by Force Therapeutics allows for remote tracking of patient recovery following TJR that allows for coordination of the patient's recovery progress with his or her care team, ${ }^{[52]}$ (2) Make it fast: Focus on asking the right questions related to a given condition rather than obtaining all possible data, and (3) Make it relevant: Show patients that you are utilizing the data in real time by addressing their responses during office visits. Implementing EMR technology that can incorporate these responses rapidly can save clinicians valuable clinic time by having responses uploaded prior to a patient visit so that results can be discussed during appointments rather than going through the questionnaire during the visit. In fact, Dr. Wagle describes this last point as the biggest determinant in whether a patient completes PROM questionnaires. ${ }^{[35]}$

\section{Conclusions}

In time with more accurate and focused collection methods of acquiring data, these two theories and practices of care will hopefully begin to see less and less divergence when it comes to applying the data derived from population health initiatives to individual patients in a patient-centered manner. Coordination between providers, governmental agencies, and payers to decide on the most beneficial metrics to record and report to central databases for the benefit of populations as well as, individual patients will be a key component in achieving this goal. In addition, utilizing new technology to streamline the collection and interpretation of data will help to reduce the burden on clinicians in obtaining and complying with reporting requirements and allow for more time and resources towards patient care.

\section{CONFlicts OF INTEREST Disclosure}

The authors declare they have no conflicts of interest.

\section{REFERENCES}

[1] Blackstone EA, Fuhr Jr JP. Redefining Health Care: Creating Value Based Competition on Results: Michael E. Porter and Elizabeth Olmsted Teisberg, Boston, MA: Harvard Business School Press;
2006. 506 p. Boston: Springer; 2007. 491-501 p.

[2] All-Cause Admissions and Readmissions Measures. National Quality Forum: Department of Health and Human Services. 2014.

[3] Zeppieri G Jr., George SZ. Patient-defined desired outcome, success 
criteria, and expectation in outpatient physical therapy: a longitudinal assessment. Health and Quality of Life Outcomes. 2017; 15(1): 29. PMid: 28143546 . https ://doi .org/10.1186/s12955-017 -0604-1

[4] Porter ME. What is Value in Health Care? New England Journal of Medicine. 2010; 363(26): 2477-81. PMid: 21142528. https: //doi.org/10.1056/NEJMp1011024

[5] Administration TSGDoHCF. Executive Summary Total Joint Replacement Episode. 2017.

[6] Harwood JL, Butler CA, Page AE. Patient-Centered Care and Population Health: Establishing Their Role in the Orthopaedic Practice. J Bone Joint Surg Am. 2016; 98(10): e40. PMid: 27194502. https://doi.org/10.2106/JBJS.15.00752

[7] Kelley T. Population-Based, Meet Patient-Centered. 2012 [cited 2017 5/21]. Available from: https://www.managedcaremag.c om/archives/2012/5/population-based-meet-patient-c entered

[8] Balint M, Ball DH, Hare ML. Training medical students in patientcentered medicine. Comprehensive Psychiatry. 1969; 10(4): 249-58. https://doi.org/10.1016/0010-440X (69) 90001-7

[9] Institute of Medicine Committee on Quality of Health Care in America, Institute of Medicine. Crossing the Quality Chasm: A New Health System for the 21st Century. Washington (DC): National Academies Press; 2001.

[10] Gerteis M, Edgman L, Daley J, et al. Through the patient's eyes: understanding and promoting patient-centered care. Journal of the American Medical Association. 1993.

[11] Zajac DP, FACOFP. Patient-centered medical home. In: Physicians ACoOF, editor. 2014. Available from: acofp.org

[12] Alluri RK, Leland H, Heckmann N. Surgical research using national databases. Annals of Translational Medicine. 2016; 4(20). PMid: 27867945. https://doi.org/10.21037/atm. 2016.10.49

[13] Sidorov J. Disease Management Care Blog. 2013 [cited 2017]. Available from: http://diseasemanagementcareblog.blog spot.com/2013/03/a-definition-of-big-data-for-hea lth.html

[14] Pugely AJ, Martin CT, Harwood J, et al. Database and Registry Research in Orthopaedic Surgery: Part I: Claims-Based Data. J Bone Joint Surg Am. 2015; 97(15): 1278-87. PMid: 26246263. https://doi.org/10.2106/JBJS.N.01260

[15] Rosenfeld RM, Shiffman RN. Clinical practice guideline development manual: A quality-driven approach for translating evidence into action. Otolaryngology-Head and Neck Surgery. 2009; 140(6 Suppl 1): S1-43.

[16] Raghupathi W, Raghupathi V. Big data analytics in healthcare: promise and potential. Health Information Science and Systems. 2014; 2: 3. PMid: 25825667. https ://doi.org/10.1186/2047 $-2501-2-3$

[17] Obermeyer Z, Emanuel EJ. Predicting the Future - Big Data, Machine Learning, and Clinical Medicine. NEJM Catalyst. 2016 [cited 2017 5/24]. Available from: http://catalyst.nejm.org/big-d ata-machine-learning-clinical-medicine/

[18] Steinberg SM, Popa MR, Michalek JA, et al. Comparison of risk adjustment methodologies in surgical quality improvement. Surgery. 2008; 144(4): 662-7; discussion -7.

[19] Naidus E. Big data in healthcare: are we close to it? 2016; 28(1): 8-10.

[20] Vigen T. Spurious Correlations: Hachette Books; 2015.

[21] Outcomes YNHHSCCf, (YNHHSC/CORE): RE. Hospital-level 30Day All-Cause Risk-Standardized Readmission Rate Following Elective Primary Total Hip Arthroplasty (THA) And/Or Total Knee
Arthroplasty (TKA). Centers for Medicare \& Medicaid Services (CMS). 2012.

[22] Topaz M, Shafran-Topaz L, Bowles KH. ICD-9 to ICD-10: Evolution, Revolution, and Current Debates in the United States. Perspectives in Health Information Management. 2013; 10.

[23] Fracture at wrist and hand level. 2016 [updated 10/1/2016; cited 2017 5/27]. Available from: http://www.icd10data.com/ICD10CM/ Codes/S00-T88/S60-S69/S62/S62.1-

[24] DesHarnais SI, Forthman MT, Homa-Lowry JM, et al. Risk-adjusted clinical quality indicators: indices for measuring and monitoring rates of mortality, complications, and readmissions. Quality management in health care. 2000; 9(1): 14-22. PMid: 11185878 https://doi.org/10.1097/00019514-200009010-00003

[25] Services CfMaM. Bluebrint for the CMS Measures Management System. 2017. Available from: cms. gov

[26] Quality Measures. Center for Medicare and Medicaid Services; 2017 [cited 2017 5/27]. Available from: https://qpp.cms.gov/meas ures/quality

[27] Bakhsh W, Mesfin A. Online ratings of orthopedic surgeons: analysis of 2185 reviews. American Journal of Orthopedics. 2014; 43(8): 359-63. PMid: 25136868.

[28] Arlene B. Harnessing the Power of Data: How AHRQ Is Catalyzing Transformation in Health Care. Agency for Healthcare Research and Quality. 2015 [cited 2017 5/21]. Available from: https://www.ahrq.gov/research/findings/factshe ets/informatic/databrief/index.html

[29] D'Apuzzo MR, Novicoff WM, Browne JA. The John Insall Award: Morbid obesity independently impacts complications, mortality, and resource use after TKA. Clinical Orthopaedics and Related Research. 2015; 473(1): 57-63. PMid: 24818736. https://doi.org/10.1 007/s11999-014-3668-9

[30] Harris AH, Bowe TR, Gupta S, et al. Hemoglobin A1C as a marker for surgical risk in diabetic patients undergoing total joint arthroplasty. The Journal of Arthroplasty. 2013; 28(8 Suppl): 25-9. PMid: 23910511. https://doi.org/10.1016/j.arth.2013.03.033

[31] Duchman KR, Gao Y, Pugely AJ, et al. The Effect of Smoking on Short-Term Complications Following Total Hip and Knee Arthroplasty. J Bone Joint Surg Am. 2015; 97(13): 1049-58. PMid: 26135071. https://doi.org/10.2106/JBJS.N.01016

[32] Muthuri SG, Hui M, Doherty M, et al. What if we prevent obesity? Risk reduction in knee osteoarthritis estimated through a metaanalysis of observational studies. Arthritis Care \& Research. 2011; 63(7): 982-90. PMid: 21425246. https : //doi .org/10.1002/ac r. 20464

[33] What is Osteoporosis and What Causes It? National Osteoporosis Foundation. [cited 2017 5/22]. Available from: https://www. nof . org/patients/what-is-osteoporosis/

[34] Osteoarthritis National Institute of Health Fact Sheets: National Institue of Health. [cited 2017 5/22]. Available from: https://report.nih.gov/nihfactsheets/ViewFactSheet . aspx?csid=55

[35] Wagle NW. Implementing Patient-Reported Outcome Measures NEJM Catalyst: NEJM Catalyst. 2016 [cited 2017 5/11]. Available from: http://catalyst.nejm.org/implementing-pro ms-patient-reported-outcome-measures/

[36] Deshpande PR, Rajan S, Sudeepthi BL, et al. Patient-reported outcomes: A new era in clinical research. Perspectives in Clinical Research. 2011; 2(4): 137-44. PMid: 22145124. https://doi.org/ 10.4103/2229-3485.86879

[37] Schonberg MA, Marcantonio ER, Hamel MB. Perceptions of Physician Recommendations for Joint Replacement Surgery in Older Patients with Severe Hip or Knee Osteoarthritis. Journal of the 
American Geriatrics Society. 2009; 57(1): 82-8. PMid: 19054184 https://doi.org/10.1111/j.1532-5415.2008.02082.x

[38] Boden SD, Smith BT, Handley M. Population Health Management: Is There Any Role for Orthopaedics?: An AOA Critical Issues Symposium. J Bone Joint Surg Am. 2017; 99(9): e45. PMid: 28463927. https://doi.org/10.2106/JBJS.16.00875

[39] Byers M. EHR integration: Keeping patients at the center of care. Healthcare IT News. 2015 [cited 2017 5/28]. Available from: http://www.healthcareitnews.com/blog/ehr-integ ration-keeping-patients-center-care

[40] Krieger N. Who and What is a "Population"? Historical. The Milbank Quarterly. 2012; 90(4): 634-81. PMid: 23216426. https: //doi.org/10.1111/j.1468-0009.2012.00678.x

[41] Abdou CM, Fingerhut AW, Jackson JS, et al. Healthcare Stereotype Threat in Older Adults in the Health and Retirement Study. American Journal of Preventive Medicine. 2016; 50(2): 191-8. PMid: 26497263. https://doi.org/10.1016/j.amepre.2015.07.034

[42] Health promotion and disease prevention through population-based interventions, including action to address social determinants and health inequity. World Health Organization. [cited 2017 5/28]. Available from: http://www.emro. who.int/about-who/public-health-f unctions/health-promotion-disease-prevention.html

[43] HATCH H. Population Health Isn't Working Out Quite the Way They Said It Would. What's Going On? 2017 [updated 2/20/2017; cited 2017 5/19]. Available from: http://thehealthcareblo g.com/blog/2017/02/20/personal-health-vs-populatio n-health/

[44] Eddy DM. Designing a practice policy: Standards, guidelines, and options. JAMA. 1990; 263(22): 3077-84. PMid: 2342221. https://doi.org/10.1001/jama.1990.03440220105041

[45] Peckham C. Medscape Physician Compensation Report. 2016.
[46] Sinsky C, Colligan L, Li L, et al. Allocation of physician time in ambulatory practice: A time and motion study in 4 specialties. Annals of Internal Medicine. 2016; 165(11): 753-60. PMid: 27595430. https://doi.org/10.7326/M16-0961

[47] Goldsmith LJ, Suryaprakash N, Randall E, et al. The importance of informational, clinical and personal support in patient experience with total knee replacement: a qualitative investigation. BMC Musculoskelet Disord. 2017; 18(1): 127. PMid: 28340610. https : //doi.org/10.1186/s12891-017-1474-8

[48] What is Hospital Compare? 2017 [cited 2017 5/28]. Available from: https://www.medicare.gov/hospitalcompare/About /What-Is-HOS.html

[49] Dundon JM, Bosco J, Slover J, et al. Improvement in Total Joint Replacement Quality Metrics: Year One Versus Year Three of the Bundled Payments for Care Improvement Initiative. J Bone Joint Surg Am. 2016; 98(23): 1949-53. PMid: 27926675. https : //doi.org/10.2106/JBJS.16.00523

[50] Rolfson O, Bohm E, Franklin P, et al. Patient-reported outcome measures in arthroplasty registries: Report of the Patient-Reported Outcome Measures Working Group of the International Society of Arthroplasty RegistriesPart II. Recommendations for selection, administration, and analysis. Acta Orthopaedica. 2016; 87(Suppl 1): 9-23. PMid: 27228230. https://doi.org/10.1080/17453674 .2016 .1181816

[51] Ayes D. Successful use of patient reported outcomes in bundled patient contracts. 2016 [cited 2017 5/20]. Available from: http://www . beckershospitalreview . com/finance/succ essful-use-of-patient-reported-outcomes-in-bundled -patient-contracts.html

[52] Dolan B. Force Therapeutics raises $\$ 2.6 \mathrm{M}$ to help hospitals take on CJR, bundled payments. 2016 [cited 2017 5/29]. Available from: http://www . mobihealthnews. com/content/force-thera peutics-raises-26m-help-hospitals-take-cjr-bundled -payments 